\title{
OROBANCHE L. (OROBANCHACEAE) SECT. TRIONYCHON WALLR., EN ANDALUCÍA II: OROBANCHE ROSMARINA BECK.
}

\author{
Antonio J. PUJADAS SALVÀ, Diego RUBIALES OLMEDO \\ y Mónica LÓPEZ MARTÍNEZ
}

\begin{abstract}
RESUMEN. Orobanche L. (Orobanchaceae) sect. Trionychon Wallr., en Andalucia II: Orobanche rosmarina Beck. Se indica la presencia de Orobanche rosmarina Beck en Andalucía. Aportamos la descripción de los ejemplares andaluces, su corología y habitat. Se incluyen unas nuevas claves de determinación para las especies andaluzas de la sect Trionychon.
\end{abstract}

Palabras clave. Orobanchaceae, Orobanche, jopo, parásito, Andalucía.

ABSTRACT. Orobanche L. (Orobanchaceae) sect. Trionychon Wallr, in Andalusia II: Orobanche rosmarina Beck. The presence of Orobanche rosmarina Beck in Andalusia is noted. Description, chorology and habitat of the Andalusian specimens is given. New identification keys are added.

Key words. Orobanchaceae, Orobanche, broomrape, parasite, Andalusia.

\section{INTRODUCCIÓN}

Beck-Mannagetta en Ginzberger (1921: 243) publicó el nombre de Orobanche rosmarina. Éste ha sido lectotipificado recientemente por Foley (2001a: 231) basándose en las recolecciones realizadas por Welwitsch en Estremadura (Portugal) y que en su material de exsiccata denominó como Trionychium Rosmarinum (Welwitsch, Flora Lusitanica, Sect. II. No. 779, Annis 1848$50)$.

Guimarães (1904: 79) indica su presencia en Portugal con el nombre de $O$. mutelii var. stenosiphon Beck, basado en el material de Welwitsch y en sus propias recolecciones en Serra da Arrábida.
Posteriormente Chater \& Webb (1972: 288) la reconocen como especie algo dudosa situándola como próxima a $O$. lavandulacea Rchb. y a O. nana (Reut.) Beck. También Greuter et al. (1989: 262) resaltan su status taxonómico dudoso con un interrogante (?). Foley (2001b: 40) indica su presencia en Estremadura (Portugal) y en Ciudad Real (España), aportando sólo una referencia bibliográfica para Baleares. No existen otras referencias fiables para esta planta ya que las citas de $O$. mutelii var. stenosiphon Beck o de $O$. rosmarina de otras zonas del área mediterránea, de acuerdo con Foley (2001a: 232; 2001b: 42), son probablemente erróneas.

Por lo tanto se trata de una especie rara, 
mal conocida y muy escasamente representada en los herbarios y que apenas se ha vuelto a encontrar en la Península Ibérica desde las recolecciones de Welwitsch y Guimarães, sólo hay constancia de un testimono para la provincia de Ciudad Real (MA 596845) identificado por Foley en 2001.

Ha sido localizada en Sierra Morena, en las provincias de Córdoba y Sevilla. Se trata de una nueva especie para el territorio andaluz y que no habíamos considerado en nuestro anterior trabajo sobre las especies de la sección Trionychon en Andalucía (Pujadas Salvà, 2000) ni en nuestra publicación sobre Orobanchaceae en la Península Ibérica e Islas Baleares (Pujadas Salvà, 2002).

\section{MATERIAL Y MÉTODO}

El material analizado ha sido recolectado en nuestras habituales prospecciones botánicas. Las plantas se han contrastado con el material original de Welwitsch (LISU) para asegurarnos de su precisa identificación. Como metodología seguimos la expuesta en Pujadas Salvà (2000: 94).

Se ha realizado una revisión crítica de los especímenes conservados en los herbarios ABH, ARAN, ALME, BC, BCCBCF, COA, COFC, COI, "Colegio La Salle de Almería", "Col.legi Oficial de Farmacèutics de les Illes Balears", GDAGDAC, HJBS, "Herbarium OrellCasanovas", JACA, LEB, LISE, LISI, LISU, LOU, MA, MAF, MGC, MPU, MUB, SALA, SANT, SEV, VAB, VAL y UIB.

\section{RESULTADOS}

Orobanche rosmarina Beck in Feddes Repert. 18: 33 (1922)
Phelypaea rosmarinii Welw. ex Nym., Consp. Fl. Eur. 557 (1881), nom. nud. Trionychon rosmarinum Welw., Fl. Lusit. Exsicc., Sect. II, n ${ }^{\circ} 779$, in sched.

Ic.: Foley (2001b: 41)

Planta de (7)8-16 cm. Tallo de 2-5(6) $\mathrm{mm}$ de diámetro en su mitad, apenas engrosado en la base, de 2-7(12) $\mathrm{mm}$ de diámetro, rara vez ligeramente bulbiforme, tallo simple a veces ramificado desde la base, glabro o glabrescente, blanquecino, frecuentemente la parte superior ligeramente teñida de morado, ocráceo o castaño en seco; eje de la inflorescencia densa y cortamente pubescente glandular (con pelos glandulíferos incoloros), de color blanquecino teñido de morado, intensamente azul-morado oscuro en seco. El tallo se encuentra por debajo del nivel del suelo hasta la inflorescencia, incluso es frecuente que algunos botones florales se encuentren también enterrados. Hojas basales y superiores 7-10 × 4-6 mm, ovadas a deltoideas, esparcidas, glabras o subglabras. Inflorescencia de 6-11 x 1,5-2,1 cm, densa, a veces algo laxa en la base, cilíndrica, con el ápice redondeado en las inflorescencias maduras (cónico al inicio de la antesis). Flores sésiles o a veces las inferiores con pedicelos de hasta $4 \mathrm{~mm}$. Brácteas 7-10 x 4,5-6 mm, menores que el cáliz, igualando la mitad del tubo de la corola, ovadas a ampliamente lanceoladas, con pelos glandulíferos de glándula incolora o anaranjada, blanquecinas teñidas muy ligeramente de morado, de color azulmorado oscuro en seco. Bractéolas 6-7 x 0,9$1,4(1,6) \mathrm{mm}$, de lineares a estrechamente lanceoladas, blanquecinas muy débilmente teñidas de morado, morado intenso en seco (azul purpúreo). Cáliz 8-11 mm, con 4 dientes triangulares, igualando o a veces hasta 1,5 veces su tubo, frecuentemente un 
quinto diente adaxial de tamaño reducido, cáliz con los nervios marcados, frecuentemente los segmentos del cáliz están superpuestos plegados longitudinalmente, uno sobre otro, en su base, cáliz de color blanquecino, teñido muy débilmente de morado, azul morado intenso en seco. Corola 12-15(16) $\mathrm{mm}$, erecto patente a veces erecta, recta a uniforme y suavemente curvada, de base algo hinchada estrechándose ligeramente por encima de la inserción de los filamentos, de 1,5-2,4 $\mathrm{mm}$ de ancho, y tubular ligeramente infundibuliforme en el ápice, de 4-5 $\mathrm{mm}$ de ancho en la garganta, cortamente pubescente glandular, con pelos incoloros, con la glándula anaranjada o amarillenta en seco, corola de base blanca, blanquecina teñida ligeramente de morado y con los nervios de color morado más intenso por encima de la inserción de los filamentos, ápice de los lóbulos de color azul, labios de color azul morado en seco. Labio superior bilobado, con los lóbulos +/agudos, profundamente emarginado, el inferior con los lóbulos subiguales, ovados a triangulares, agudos, de margen glabro o glabrescente (con algún pelo glandulífero disperso muy corto de 0,02-0,04 mm). Filamentos insertos subhorizontalmente, los adaxiales a 4-6 mm, los abaxiales a 3,5-5 $\mathrm{mm}$, glabros a veces subglabros (escasos pelos glandulares por debajo de las anteras, blancos; anteras 1,2-1,4 $\mathrm{mm}$ (apículo 0,2 $\mathrm{mm}$ ), oblongas, blancas o amarillentas, blancas en seco, glabras. Ovario glabro, blanco teñido de morado a veces completamente morado. Estilo glabro, morado. Estigma discoideo, escasamente bilobado, \pm aplanado, blanco.

Habita en claros de encinar, generalmente bien conservado, en el piso termomediterráneo, sobre Rosmarinus officinalis. 200-800 m. En Andalucía, se encuentra en la Provincia corológica Luso-
Extremadurense, Sector MariánicoMonchiquense (Rivas Martínez, 1982). Su situación geográfica corresponde a la comarca natural de Sierra Norte (Valdés et al., 1987).

\section{MATERIAL ESTUDIADO}

ESPAÑA: CIUDAD REAL: Solana del Pino, valle del Arroyo del Nacedero, $680 \mathrm{~m}$, jarales sobre suelos profundos, 30SUH9860, 26-IV1997, R. García Río, MA 596845. CÓRDOBA: Obejo, Río Guadalbarbo, cerca del Cortijo del Cura, 30SUH4117, $290 \mathrm{~m}$, sobre Rosmarinus officinalis, con Orobanche latisquama, 18-V2001, D. Rubiales \& A. Pujadas, COA 31029. SEVILla: El Real de la Jara, Sierra del Pimpollar, Cortijo de las Águilas, 30SQC5605, 740 m, 19-VI-1997, J.M. Delgado \& M. López, COA 23756. El Real de la Jara, Sierra del Pimpollar, Cortijo de las Águilas, 30SQC5605, 740 m, sobre Rosmarinus ?,9-VII-1997, M. López \& A. Pujadas, COA 23755.

PORTUGAL: ESTREMADURA: Ad radices Rosmar. off. in ... Serra da Arrábida, rarior, rarius adhuc radices'Quercus humilis, in Serra de Montejunto, ubi etiam ad'Rosmarinus..., flor Maio et Junio, Welwitsch, LISU P34461 [sub'Phelipaea (Trionychon) Rosmarini Welw.]; Serra da Arrábida, Casal da Pimenta, sobre Rosmarinus, IV-1903, A. Guimarães, $\mathrm{n}^{\circ} 2270$, LISU P 34459 [sub Orobanche ramosa b. Muteli (F. Schultz) var. stenosiphon Beck].

O. rosmarina se diferencia claramente del resto de las especies de la sección Trionychon por su corola con el margen glabro o subglabro, y frecuentemente enterrado hasta la inflorescencia, incluso los botones florales inferiores están, a veces, también enterrados.

Chater \& Webb (1972) señalan ciertas analogías con $O$. lavandulacea por la coloración azul-morada profunda que presentan sus inflorescencias al secarse, pero de la que se separa, además, por su menor porte, tallos simples raramente ramificados, hojas más cortas, inflorescencia 
generalmente más corta, cáliz de dientes triangulares, corola de tamaño menor, blanquecina teñida de púrpura en fresco y anteras más cortas. Los mismos autores indican parecido con $O$. nana principalmente por su hábito y de la que se diferencia, además, por tener el cáliz más largo, con dientes triangulares sin estar largamente acuminados y los filamentos estaminales glabros o a veces subglabros.

Las citas para el norte y este de la Península Ibérica de Carlón et al. (2002: 10; 2003: 9-10, 14) requieren ser confirmadas. Carlón et al. (2003: 9, 14) incluso manifiestan ciertas divergencias entre las plantas estudiadas y la descripción de Foley (2001b: 40) y además aportan una fotografía de una «Orobanche rosmarina» con unos caracteres muy distintos con respecto al taxon arriba descrito y con respecto al icono de Foley (2001b: 41) como son el tipo de inflorescencia (corta y muy densa), forma de los dientes del cáliz (alesnado), indumento de la corola (densamente pubescente, margen claramente ciliado).

Las citas de Beckett (1993: 148) para Mallorca también requieren confirmación ya que la planta dibujada por la autora presenta un indumento que no es el típico de Orobanche rosmarina s. str.

\section{Claves para la Sect. Trionychon}

Aportamos unas nuevas claves de identificación para la sección Trionychon que sustituyen a las publicadas anteriormente por Pujadas Salvà (2000) para Andalucía.

\section{a) para material fresco}

1. Cáliz (13)16-21 mm. Corola de (18)21-30 mm, azul violeta intenso

o. arenaria Cáliz 4-16 mm. Corola de 12-21 mm blanquecina a azul intenso en el ápice .......... 2

2. Cáliz (10)13-16 mm, con dientes 1,5-3 veces la longitud del tubo O. schultzii Cáliz 4-12 mm, con dientes menores que 1,5 veces la longitud del tubo

3. Margen de la corola glabro o subglabro. Filamentos glabros o subglabros. Frecuentemente tallo completamente enterrado, sólo la inflorescencia es aérea.....

O. rosmarina

Margen de la corola ciliado o brevemente pubescente glandular. Filamentos pelosos a glabros. Tallo aéreo visible así como la inflorescencia .. 4

4. Cáliz 4-7,5 mm. Corola 12-16(17) mm ........ 5 Cáliz 7-12 mm. Corola (15)17-22 mm ....... 6

5. Planta $17-35 \mathrm{~cm}$, tallo muy ramificado desde la base. Inflorescencia 10-25 cm, laxa. Dientes del cáliz triangulares brevemente acuminados. Corola blanquecina o azul pálido en el ápice

O. ramosa

Planta 8-20 cm, tallo simple o ramificado. Inflorescencia 3,5-7(8) cm densa a veces laxa en la base. Dientes del cáliz lanceolados a triangulares largamente acuminados de ápice subfiliforme. Corola azul brillante en el ápice, a veces azul oscuro

o. nana

6. Planta (7)12-20 cm. Inflorescencia $(2,5) 5-8 \mathrm{~cm}$, densa. Cáliz 7-10 mm. Corola de azul pálido a azul brillante en el ápice

O. mutelii Planta 19-53 cm. Inflorescencia 5-47 cm, laxa al menos en la base. Caliz 8-12 mm. Corola azul oscuro o azul o morado pálido 7

7. Tallo ramificado. Inflorescencia (12)19-47 cm. Corola azul oscuro intenso ... O. lavandulacea Tallo simple. Inflorescencia 5-9 cm. Corola azul pálido con venas longitudinales más oscuras

O. purpurea

\section{b) para material de herbario}

1. Dientes del cáliz 1,5-3 veces la longitud del tubo O. schultzii Dientes del cáliz menores que 1,5 veces la longitud del tubo

2. Cáliz (13)16-21 mm. Corola de (18)21-30 mm O. arenaria Cáliz menor de $12 \mathrm{~mm}$. Corola menor de 21 $\mathrm{mm}$

3. Cáliz 4-7,5 mm. Corola 12-16(17) $\mathrm{mm}$........ 4 Cáliz 7-12 mm. Corola (15)17-22 mm .......... 6

4. Planta $17-35 \mathrm{~cm}$, muy ramificada desde la base. Inflorescencia $10-25 \mathrm{~cm}$, laxa. Cáliz 4-6 mm

O. ramosa 
Planta 8-20 cm, simple o ramificada. Inflorescencia 3,5-11 cm, densa, a veces algo laxa en la base. Cáliz 5-11 mm .

5. Brácteas 2,5-3,5 mm de ancho. Cáliz 5-7,5 mm con dientes lanceolados de ápices acuminados filiformes. Filamentos estaminales pelosos ...

o. nana

Brácteas 4,5-6 mm de ancho. Cáliz 8-11 mm con dientes triangulares-lanceolados. Filamentos estaminales glabros o subglabros

O. rosmarina

6. Planta (7)12-20 cm. Anteras 1,3-1,6 mm O. mutelii

Planta $19-53 \mathrm{~cm}$. Anteras $(1,2) 1,5-2 \mathrm{~mm}$..... 7

7. Planta $19-29 \mathrm{~cm}$. Tallo simple. Inflorescencia \pm laxa O. purpurea Planta (25)33-53 cm. Tallo ramificado. Inflorescencia densa O. lavandulacea

AGRADECIMIENTOS. A los conservadores y personal de los herbarios ABH, ARAN, ALME, BC, BCC-BCF, COFC, COI, "Colegio La Salle de Almería, "Col.legi Oficial de Farmacèutics de les Illes Balears", GDA-GDAC, HJBS, "Herbarium Orell-Casanovas", JACA, LEB, LISE, LISI, LISU, LOU, MA, MAF, MGC, MPU, MUB, SALA, SANT, SEV, VAB, VAL y UIB por las facilidades en la consulta del material. A Gonzalo Nieto Feliner y a Félix Muñoz Garmendía por su ayuda en los aspectos nomenclaturales.

\section{BIBLIOGRAFÍA}

BECKETT, E. -1993-Illustrated Flora of Mallorca. Palma de Mallorca.

CARLÓN, L., G. GÓMEZ CASARES, M. LAÍNZ, G. MORENO MORAL y Ó. SÁNCHEZ PEDRAJA -2002-A propósito de algunas Orobanche (Orobanchaceae) del noroeste peninsular y de su tratamiento en Flora iberica vol. XIV (2001). Documentos Jard. Bot. Atlántico (Gijón) 1: 1-44.

CARLÓN, L., G. GÓMEZ CASARES, M. LAIINZ, G. MORENO MORAL y Ó. SÁNCHEZ PEDRAJA -2003- Más, a propósito de algunas Orobanche (Orobanchaceae) del norte y este de la Península Ibérica. Documentos Jard. Bot. Atlántico (Gijón) 2: 1-45.
CHATER, A. O. \& D.A. WEBB -1972Orobanche, in: Tutin T.G., V.H. Heywood, N.A. Burges, D.M. Moore, D.H. Valentine, S.M. Walters \& D.A. Webb (Eds.), Flora Europaea 3: 286-293. Cambridge.

FOLEY, M.J.Y. -2001a- Orobanchaceae in the "Flora Iberica" area: new taxa, excluded taxa, and typification. Anales Jard. Bot. Madrid 58(2): 223-233.

FOLEY, M.J.Y. -2001b- Orobanche L., in: Paiva J., F. Sales, I.C. Hedge, C. Aedo, J.J. Aldasoro, S. Castroviejo, A. Herrero \& M. Velayos (Eds.), Flora iberica 14: 32-72. Madrid.

GINZBERGER, A. von -1921- Beitrag zur Kenntnis der Flora der Scoglien und kleineren Inseln Süd-Dalmatiens. Oesterr. Bot. Z. 70 (9-12): 233-248.

GREUTER, W.R., H.M. BURDET \& G. LONG 1989-Med-Checklist. Vol.4. (LauraceaeRhamnaceae). Genève.

GUIMARÃES, J.A. -1903- Monographia das Orobanchaceas. Brotéria 3: 1-188.

PUJADAS SALVÀ, A.J. -2000- Orobanche L. (Orobanchaceae) sect. Trionychon Wallr., en Andalucía. Acta Bot. Malacitana 25: 93-106.

PUJADAS SALVÀ, A.J. -2002- Orobanche L., in López-Sáez J.A., P. Catalán \& Ll. Sáez (Eds.), Plantas Parásitas de la Península Ibérica e Islas Baleares: 348-440. Madrid, Barcelona \& México.

RIVAS MARTÍNEZ, S. -1982- Mapa de las series de vegetación de Madrid. Diputación Provincial de Madrid.

VALDÉS, B., S. TALAVERA \& E. FERNÁNDEZ-GALIANO (Eds.) -1987Flora Vascular de Andalucía Occidental, Vol. 1. Barcelona.

Aceptado para su publicación en abril de 2005

Dirección de los autores. A. J. PUJADAS SALVÀ: Departamento de Ciencias y Recursos Agrícolas y Forestales. Universidad de Córdoba. Apdo. 3048. E-14080 Córdoba. E-mail: cr1pusaa@uco.es. D. RUBIALES OLMEDO: Instituto de Agricultura Sostenible - CSIC, Apdo. 4084. E-14080 Córdoba. M. LÓPEZ MARTÍNEZ: Jardín Botánico de Córdoba. Avda. Linneo s/n. E-14004 Córdoba. 
